\title{
The high level of adherence to personal protective equipment in health care workers efficiently protects them from COVID-19 infection
}

\author{
Malihe Zangoue ${ }^{\mathrm{a}}$, Hamidreza Safari ${ }^{\mathrm{b}}$, Simon G. Royce ${ }^{\mathrm{c}}$, Alireza Zangooie ${ }^{\mathrm{d}}$, Hadis Rezapour ${ }^{\mathrm{d}}$, \\ Amirsadra Zangouei ${ }^{\mathrm{e}}$ and Mohammad Fereidouni ${ }^{\mathrm{f}, *}$ \\ ${ }^{a}$ Department of Anesthesiology and Intensive Care, Faculty of Medicine, Birjand University of Medical Sciences, \\ Birjand, Iran \\ ${ }^{\mathrm{b}}$ Departement of Immunology, Torbat Jam Faculty of Medical Sciences, Torbat Jam, Iran \\ ${ }^{\mathrm{c}}$ Department of Pharmacology, Monash University, Clayton, Australia \\ ${ }^{\mathrm{d}}$ Student Research Committee, Faculty of Medicine, Birjand University of Medical Sciences, Birjand, Iran \\ ${ }^{\mathrm{e}}$ Student Research Committee, Faculty of Medicine, Mashhad University of Medical Sciences, Mashhad, Iran \\ ${ }^{\mathrm{f}}$ Cellular and Molecular Research Center, Birjand University of Medical Sciences, Birjand, Iran
}

\begin{abstract}
.
BACKGROUND: The first case of coronavirus disease 2019 (COVID-19) was reported in December 2019 in Wuhan, China. Healthcare workers (HCWs) are at high risk of acquiring and spreading the COVID-19 infection; using personal protective equipment (PPE) reduces the risk of COVID-19 infection in HCWs.

OBJECTIVE: Our study aimed to investigate the seroprevalence of COVID-19 IgG, IgM antibodies among HCWs as well as identifying the factors associated with this seroprevalence.

METHODS: This cross-sectional study was performed from July to August 2020 on healthcare workers at two COVID-19 referral hospitals of Birjand University of Medical Sciences. The level of COVID-19 IgG and IgM antibodies in sera was measured by commercial qualitative ELISA kits.

RESULTS: In total, 192 individuals participated in the study: physicians (31.25\%), nurses (30.2\%). 84.2\% of participants had contact with confirmed COVID-19 cases and among them $42.9 \%$ of had close contact with COVID-19 patients for more than 3 months, and $31 \%$ reported close contact with more than 50 confirmed COVID-19 cases. Mask and gloves were the most frequently used personal protective equipment (PPE) with $92.4 \%$ and $77.2 \%$ of usage.

CONCLUSIONS: The results of the current study showed high level of adherence to the use of PPE among HCWs as well as very low prevalence of seropositivity for of COVID-19 antibodies, hence confirming the effectiveness of PPE in protecting HCWs among COVVID-19 and possibly any other similar infections.
\end{abstract}

Keywords: COVID-19, PPE, HCW, seroprevalence, antibody

*Address for correspondence: Mohammad Fereidouni, Cellular and Molecular Research Center, Birjand University of Medical Sciences, Birjand, Iran. E-mail: dr.m.fereidouni@gmail.com. 


\section{Introduction}

During the last 13 months from the detection of the first case of coronavirus disease 2019 (COVID- 19) in December 2019 in Wuhan, China, the COVID-19 pandemic has caused more than 93 million documented infections and over 2 million deaths [1]. In spite of all efforts and trials, no cure or highly effective treatment is available. With regard to vaccines, although several types have been developed and vaccination programs started in many countries, coverage of vaccination in global scale is still low and there is conflicting data about the level and duration of vaccines' protection particularly against new variants of SARS-2.

The extremely contagious nature of the severe acute respiratory syndrome coronavirus 2 (SARS$\mathrm{CoV}-2$ ) and prolonged incubation time contributes toarise in the number of a symptom a ticcarriers who may not take suitable precautions and possibly spread the virus [2]. For example, in a study performed in Italy, $44 \%$ of cases that had positive laboratory results didn't have any symptoms [3].

On the other hand, hospital-associated spread is regarded as a key route for virus transmission, and healthcare workers (HCWs) are at high risk for acquiring and spreading the infection [4]. Several surveys reported variable rate of COVID-19 infection among HCWs, around 4.2\% in Oman and China $[5,6]$ to $9 \%$ and even $17.8 \%$ in Italy and the USA respectively $[7,8]$.

Asymptomatic patients or those with unusual COVID-19 symptoms create a considerable hazard for HCWs, furthermore infected HCWs can transmit the infection to the patients as well [9]. Considering the fundamental role of HCWs in the management and treatment of COVID-19 patients, protecting them from COVID-19 is of fundamental importance. From the start of the pandemic, there has been a huge amount of debate about the optimal way of protection particularly for HCWs who have frequent close contact with infected patients. During the early phase of the pandemic, the role of personal protective equipment (PPE) in the provision of adequate protection for HCWs was not well-understood, and it was uncertain whether the data regarding the protective effect of N95 respirators, surgical masks, and hand hygiene in other respiratory virus infections $[10,11]$ would apply to COVID-19 as well. As we moved forward, a growing body of evidence indicated that respiratory droplets containing viable virus constitute a key transmission route for COVID-19, highlighting the preventive efficacy of PPE, especially face masks and respirators [12-14].

Although real time reverse transcriptase polymerase chain reaction (RT-PCR) is considered the gold standard method for diagnosing COVID-19, serology for COVID-19 antibodies is more useful in seroepidemiological studies and for checking the history of exposure particularly in asymptomatic cases [15]. The antibody response in HCWs has been studied in various reports with different rates, depending on the region, the time of the study, symptomatic status, and type of employee. Seroprevalence rates among HCWs has varied from $0.7 \%$ to $11 \%$ in different studies [16-20]. Notably, some of these investigations revealed the seropositivity rate among asymptomatic cases ranges from $38 \%$ to $48 \%$ [20-22]. Evaluating the presence of anti-SARS-CoV2 in healthcare workers gives evidence about the effectiveness of PPEs usage in protection of health care workers [21]. Eventually, surveillance outcomes for asymptomatic HCWs are also helpful to decrease the labor shortage due to excessive quarantine, to distinguish atypical, mild, or asymptomatic cases, and in protecting all healthcare employees.

Regarding the important role of HCWs in controlling and treatment of pandemic and the role of PPE in protection against COVID-19, the aims of the current study was to evaluate the adherence of HCWs to $t$ proper use of PPEs and the seroprevalence of SARS-CoV-2 antibodies among a group of HCWs in COVID-19 referral hospitals at Birjand city, Iran.

\section{Materials and methods}

This cross-sectional study, performed from July to August 2020, on healthcare workers at two COVID19 referral hospitals of Birjand University of Medical Sciences. The rate of exposure to COVID-19 patients inside and outside the hospitals, the level of adherence to use of PPE among health care workers as well as demographic information was assessed by a questionnaire which was originated from the WHO questionnaire with some modification. The study was approved by the ethics committee of Birjand University of Medical Sciences (ir.bums.REC.1399.109) and all participants signed an informed consent form. All collected questionnaires were checked and five milliliters of venous blood was taken from participants who filled the questionnaires properly and agreed to donate blood samples. Serum was separated by centrifugation and kept at $-20^{\circ} \mathrm{C}$ until analysis. 
Table 1

Proportion of different participants based on their occupation

\begin{tabular}{lc}
\hline Occupation & Number $(\%)$ \\
\hline Physicians & $60(31.25)$ \\
Nurses & $58(30.2)$ \\
Radiology staff & $15(7.8)$ \\
Service staff & $10(5.2)$ \\
Reception staff & $7(3.65)$ \\
Laboratory staff & $9(4.7)$ \\
Other & $33(17.2)$ \\
Total & $192(100)$ \\
\hline
\end{tabular}

The level of COVID-19 IgG and IgM antibodies in sera was measured by commercial qualitative ELISA kits (Pishtaz Teb Company, Tehran, Iran). The sensitivity and specificity of the assay were $85.4 \%$ and $99.4 \%$ for IgM and $94.1 \%$ and $98.3 \%$ for IgG respectively. Those with positive or borderline ELISA results were re-checked.

\subsection{Statistical analysis}

The data were analyzed by Chi-square and MannWhitney $U$ test and the variables were expressed as frequency and percentage. A p-value less than 0.05 were considered as significant. SPSS 23 software was used for statistical analysis.

\section{Result}

In total, $192 \mathrm{HCWs}$ (mean age: $35.45 \pm 8.05, \mathrm{M} / \mathrm{F}$ ratio: 0.51$)$ participated in this study. Most of the participants were physicians $(31.25 \%)$ followed by nurses (30.2\%). Table 1 shows the proportion of participants based on their occupation.

$84.2 \%$ of participants had contact with confirmed COVID-19 cases and among them $42.9 \%$ of had close contact with COVID-19 patients for more than 3 months and $31 \%$ reported close contact with more than 50 confirmed COVID-19 cases. Figure 1 depicts the duration of exposure to patients among HCWs. Mask and gloves were the most frequently used PPE with $92.4 \%$ and $77.2 \%$ of usage, respectively and almost all of participants wear them always when were at works. Table 2 shows the usage of different PPE among participants.

In the case of seroprevalence, 137 participants donated blood and among them only 7 cases $(5.1 \%)$ including 2 males and 5 females were positive and 2 cases $(1.4 \%)$ had borderline results for COVID-19 IgG. None were positive for COVID-19 IgM. 71.42\%

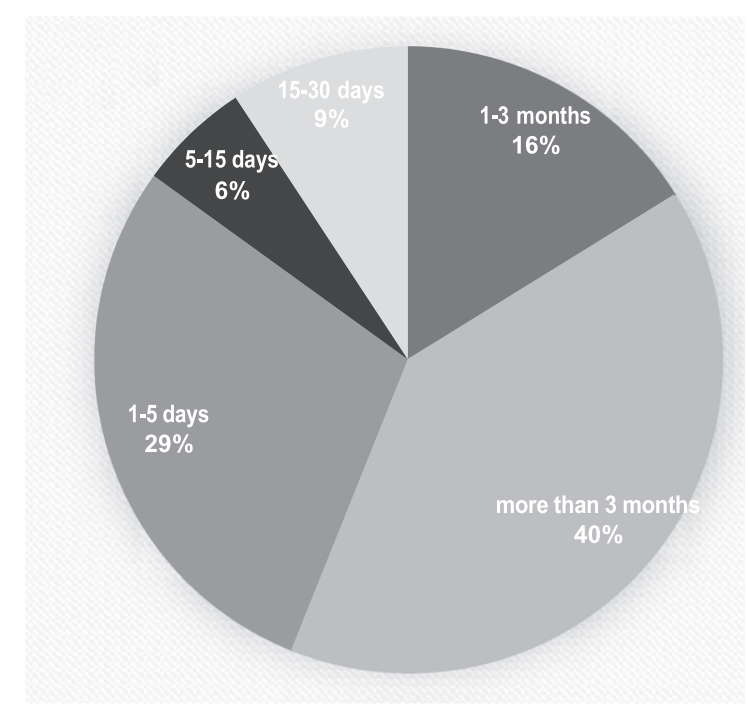

Fig. 1. Duration of close contacts with COVID-19 patients among participants.

of the individuals with positive IgG results had symptoms four weeks before filling the questionnaire. Sore throat and fatigue were the most common symptoms in IgG-positive participants. Only one of the IgGpositive individuals had an underlying disease (obesity). There was no correlation between seropositivity and occupation, rate of exposure or the level of adherence to PPE.

\section{Discussion}

Severe acute respiratory syndrome coronavirus 2 (SARS-CoV-2) is a highly contagious disease which has left many casualties particularly among HCWs around the world [22]. In spite of all efforts for treating and preventing this disease by different regimens and vaccination, using PPE remains the best protective strategy, especially for HCWs [23]. In the current we assessed the use of PPE among HCWs and the rate of seroprevalence of COVID-19.

In the present study, face masks and gloves were the most commonly used PPE. Recent studies indicated that face masks and gloves were the most commonly used PPE to protect from respiratory infections [24].

Although a loose mask does not provide complete protection from respiratory infection [25]. studies including the current study confirmed the protective effect of a mask. Our result indicates that surgical masks were a more commonly used PPE compared to N95 masks. Availability, low cost, and low side 
Table 2

Percentage of using different personal protective equipment (PPE) among HCWs

\begin{tabular}{lccccc}
\hline PPE & Percentage & \multicolumn{3}{c}{ The frequency of PPE usage (\%) } \\
\cline { 3 - 6 } & & Rarely & Occasionally & $\begin{array}{c}\text { Most of } \\
\text { the time }\end{array}$ & Always \\
\hline N95 or surgical mask & 92.4 & 0 & 1 & 38.7 & 60.3 \\
N95 mask & 64.7 & 0 & 0 & 46.3 & 53.7 \\
Surgical mask & 77.2 & 0 & 2.8 & 44.4 & 52.8 \\
Isolation gown & 72.3 & 0 & 0 & 36.8 & 63.2 \\
Gloves & 92.4 & & 1.6 & 39.7 & 58.7 \\
Shoe cover & 53.8 & 0 & 0 & 44.8 & 55.2 \\
Hair cover & 58.2 & 0 & 2.2 & 33.4 & 64.4 \\
\hline
\end{tabular}

effects of surgical masks make it more commonly used versus N95/PAPR masks [26]. Health workers who use masks have symptoms such as respiratory symptoms, pressure-related skin lesions, and dermatosis of different grade or ocular symptoms. The symptoms were higher in the healthcare staff wearing an N95/FFP2 respirator mask and this justifies the higher rate of surgical mask used [27].

Gloves are used to protect hands from blood and body fluids, including respiratory secretions [28]. Medical gloves play an essential role in the COVID19 control strategy, and The Centers for Disease Control and Prevention (CDC) and the European Center for Disease Prevention and Control (ECDC) recently recommended regular use of gloves both in the health care setting and, in the community $[29,30]$. Gloves should be worn when providing direct care for a COVID -19 case and then removed, followed by hand hygiene between COVID-19 patients. Using the same gloves for a cohort of COVID-19 cases (extended use) must not be done [26]. In contrast, The World Health Organization (WHO) has warned about the limited protective efficacy of gloves. Regular use of gloves for daily activities may lead to a false sense of protection and increased hand-to-face contact and contamination [26].

Overall, the percentage of glove use among health workers in this study was higher than in other studies, which due to the low seroprevalence of COVID-19 can indicate the correct and appropriate use of gloves among HCWs [27].

According to the protocols of the World Health Organization, the use of gloves, medical mask, gown, eye protection (goggles or face shield) and masks is recommended for Health care workers who are in direct contact with the patient, in the absence of aerosol-generating procedures; and in the presence of aerosol-generating procedures, respirator N95 or FFP2 or FFP3 standard, or equivalent masks should be used [26]. In present study, $50.86 \%$ of the partic- ipants wore 3 or more PPE, which was very similar to the protocol of the World Health Organization.

In this study seroprevalence for SARS-CoV-2 among HCWs, was low (5.1\%) while the rate of exposure to COVID-19 patients was fairly high. This prevalence was higher than other studies including the Santa Clara County, United States with approximately 3500 subjects and an IgG seroprevalence of $1.5 \%$, the Germany study on 316 with IgG seroprevalence antibody $1.6 \%$ during the weeks between 25 March and 21 April 2020 [31, 32]. Also, the $\mathrm{IgG}$ and $\operatorname{IgM}$ seroprevalence was $3.8 \%$ and $0.8 \%$ respectively among HCWs $(n=714)$ from the city of Wuhan. The IgG seropositive prevalence rate was lower $(1.3 \%)$ within the HCWs $(n=3091)$ in two nearby cities of the Hubei province and to $1.2 \%$ among $260 \mathrm{HCW}$ s from two other cities farther south of Wuhan [33]. The variety of seroprevalence in different geographic areas was consistent with the SARS-CoV-2 distribution trend in China. In contrast, several studies have examined seropositivity in healthcare workers that mostly showed a higher seropositive prevalence among healthcare workers including Spain (9.3\%), Belgium (6.4\%), and the United States (7.6\% to 8.8\%) [19, 34, 35]. Also, a higher rate of $\mathrm{HCW}$ infection was observed in a study from the United Kingdom by Keeley et al., that revealed $18 \%$ IgG positive in HCWs [36]. A study on seroprevalence in healthcare workers in Sweden revealed that the seroprevalence of $\mathrm{IgG}$ antibodies against SARS-CoV-2 was $19.1 \%$ among the 2149 healthcare workers recruited between April 14th and May 8th, 2020 [37]. Higher rates of seropositivity for COVID-19 IgG in some reports can be due to unpreparedness of the organizations, unawareness of the personnel and shortage of PPE. In addition, several factors can affect the rate of transmission including time of study, awareness of the people, local and national infection control policies, availability of PPE and so on. 
Some reports indicate that in the UK and Italy, HCWs experienced extreme situations during the COVID-19 pandemic, wearing paper face masks and plastic aprons instead of appropriate masks, and gowns $[2,38,39]$. Lack of IgM response in our study is logical as IgM usually appears during acute phase of infections and declines rapidly during time and among our participants, no one had active COVID-19 and those with positive IgG, passed the acute phase of disease and the IgM was undetectable [40]. The timing of the blood assortment and also the different early immune response can have an effect on the interpretation of the serological results. These differences in seroprevalence among studies may be attributable to several reasons, such as different study populations, different antibody test accuracy (e.g., on sensitivity and specificity), different lockdown and quarantine measures, and different dates of data collection [7]. Low seroprevalence of SARS- CoV-2 in this study might be explained by good adherence to infection prevention and control measures and appropriate use of PPE among HCWs in the Birjand hospitals.

The current investigation has some limitations. The present seroprevalence is based on a cross- sectional design and, at that point, cannot demonstrate the incidence of infection and disease in a cohort of patients; the follow-up could be vital to better assess the seroconversion in individuals exposed to contagious patients. Besides, employees were included based on volunteering to participate. Volunteers may have had more reason to think they could be positive than those who did not volunteer.

Unlike previous studies, there was no significant relationship between HCWs wearing an N95/PAPR mask and those use surgical/other masks or no mask, which may relate to the small sample size $[35,41]$.

Generally, optimal PPE is unclear yet, but the thorough utilization of PPE measures and absolute adherence to all infection prevention and control measures are crucial to reduce nosocomial transmission of SARS-CoV-2 and shortages of PPE increase the probability of a SARS-CoV-2 seropositive test in HCWs [7].

\section{Conclusion}

The results of the current study showed high level of adherence to use PPE among HCWs as well as very low prevalence of seropositivity for of COVID-19 antibodies which confirmed the effectiveness of PPE in protecting HCWs from COVID-19 and possibly other infection with similar transmission way.

\section{Acknowledgments}

The authors thank the study participants.

\section{Conflict of interest}

The authors declare no conflict of interest.

\section{References}

[1] Cortinovis M, Perico N, Remuzzi G. Long-term followup of recovered patients with COVID-19. The Lancet. 2021;397(10270):173-5

[2] Lai X, Wang M, Qin C, Tan L, Ran L, Chen D, et al. Coronavirus disease 2019 (COVID-2019) infection among health care workers and implications for prevention measures in a tertiary hospital in Wuhan, China. JAMA network open. 2020;3(5):e209666-e.

[3] Assessment RR. Coronavirus disease 2019 (COVID-19) in the EU/EEA and the UK-ninth update. European Centre for Disease Prevention and Control: Stockholm. 2020.

[4] Fusco F, Pisaturo M, Iodice V, Bellopede R, Tambaro O, Parrella G, et al. COVID-19 among healthcare workers in a specialist infectious diseases setting in Naples, Southern Italy: results of a cross-sectional surveillance study. Journal of Hospital Infection. 2020;105(4):596-600.

[5] Al Maskari Z, Al Blushi A, Khamis F, Al Tai A, Al Salmi I, Al Harthi $\mathrm{H}$, et al. Characteristics of healthcare workers infected with COVID-19: A cross-sectional observational study. International Journal of Infectious Diseases. 2021;102:32-6.

[6] Zhan M, Qin Y, Xue X, Zhu S. Death from Covid-19 of 23 health care workers in China. New England Journal of Medicine. 2020;382(23):2267-8.

[7] Galanis P, Vraka I, Fragkou D, Bilali A, Kaitelidou D. Seroprevalence of SARS-CoV-2 antibodies and associated factors in health care workers: a systematic review and metaanalysis. Journal of Hospital Infection. 2020.

[8] Livingston E, Bucher K. Coronavirus disease 2019 (COVID-19) in Italy. Jama. 2020;323(14):1335.

[9] Wang D, Hu B, Hu C, Zhu F, Liu X, Zhang J, et al. Clinical characteristics of 138 hospitalized patients with 2019 novel coronavirus-infected pneumonia in Wuhan, China. Jama. 2020;323(11):1061-9.

[10] Saunders-Hastings P, Crispo JA, Sikora L, Krewski D. Effectiveness of personal protective measures in reducing pandemic influenza transmission: A systematic review and meta-analysis. Epidemics. 2017;20:1-20.

[11] MacIntyre CR, Wang Q, Cauchemez S, Seale H, Dwyer $\mathrm{DE}$, Yang $\mathrm{P}$, et al. A cluster randomized clinical trial comparing fit-tested and non-fit-tested N95 respirators to medical masks to prevent respiratory virus infection in health care workers. Influenza and other respiratory viruses. 2011;5(3):170-9.

[12] Sommerstein R, Fux CA, Vuichard-Gysin D, Abbas M, Marschall J, Balmelli C, et al. Risk of SARS-CoV-2 transmission by aerosols, the rational use of masks, and protection of healthcare workers from COVID-19. Antimicrobial Resistance \& Infection Control. 2020;9(1):1-8. 
[13] Bartoszko JJ, Farooqi MAM, Alhazzani W, Loeb M. Medical masks vs N95 respirators for preventing COVID-19 in healthcare workers: A systematic review and meta-analysis of randomized trials. Influenza and other respiratory viruses. 2020;14(4):365-73.

[14] Matthews R, Young A. Medical masks vs N95 respirators for preventing COVID-19 in healthcare workers: A systematic review and meta-analysis of randomized trials: Bartoszko JJ, Farooqi MAM, Alhazzani W, and Loeb M. Influenza and Other Respiratory Viruses. 2020. Journal of Emergency Medicine. 2020;59(1):165-6.

[15] Kubina R, Dziedzic A. Molecular and serological tests for COVID-19 a comparative review of SARS-CoV-2 coronavirus laboratory and point-of-care diagnostics. Diagnostics. 2020;10(6):434.

[16] Brandstetter S, Roth S, Harner S, Buntrock-Döpke $\mathrm{H}$, Toncheva AA, Borchers $\mathrm{N}$, et al. Symptoms and immunoglobulin development in hospital staff exposed to a SARS-CoV-2 outbreak. Pediatric allergy and immunology. 2020;31(7):841-7.

[17] Folgueira MD, Munoz-Ruiperez C, Alonso-Lopez MA, Delgado R. SARS-CoV-2 infection in health care workers in a large public hospital in Madrid, Spain, during March 2020. medRxiv. 2020.

[18] Galan I, Velasco M, Casas ML, Goyanes MJ, RodriguezCaravaca G, Losa JE, et al. SARS-CoV-2 SEROPREVALENCE AMONG ALL WORKERS IN A TEACHING HOSPITAL IN SPAIN: UNMASKING THE RISK. MedRxiv. 2020.

[19] Garcia-Basteiro AL, Moncunill G, Tortajada M, Vidal M, Guinovart C, Jimenez A, et al. Seroprevalence of antibodies against SARS-CoV-2 among health care workers in a large Spanish reference hospital. Nature Communications. 2020;11(1):1-9.

[20] Paderno A, Fior M, Berretti G, Schreiber A, Grammatica A, Mattavelli D, et al. SARS-CoV-2 infection in health care workers: cross-sectional analysis of an otolaryngology unit. Otolaryngology-Head and Neck Surgery. 2020;163(4):6712.

[21] De Carlo A, Lo Caputo S, Paolillo C, Rosa AM, D'orsi U, De Palma M, et al. SARS-CoV-2 serological profile in healthcare professionals of a Southern Italy hospital. International Journal of Environmental Research and Public Health. 2020;17(24):9324.

[22] Rivett L, Sridhar S, Sparkes D, Routledge M, Jones NK, Forrest S, et al. Screening of healthcare workers for SARSCoV-2 highlights the role of asymptomatic carriage in COVID-19 transmission. Elife. 2020;9:e58728.

[23] Verbeek JH, Rajamaki B, Ijaz S, Sauni R, Toomey E, Blackwood B, et al. Personal protective equipment for preventing highly infectious diseases due to exposure to contaminated body fluids in healthcare staff. Cochrane Database of Systematic Reviews. 2020(4).

[24] Chughtai AA, Khan W. Use of personal protective equipment to protect against respiratory infections in Pakistan: A systematic review. Journal of Infection and Public Health. 2020;13(3):385-90.

[25] Derrick JL, Gomersall C. Protecting healthcare staff from severe acute respiratory syndrome: filtration capacity of multiple surgical masks. Journal of Hospital Infection. 2005;59(4):365-8.

[26] Organization WH. Rational use of personal protective equipment for COVID-19 and considerations during severe shortages: interim guidance, 23 December 2020. World Health Organization; 2020.
[27] Battista RA, Ferraro M, Piccioni LO, Malzanni GE, Bussi M. Personal Protective Equipment (PPE) in COVID 19 Pandemic: Related Symptoms and Adverse Reactions in Healthcare Workers and General Population. Journal of Occupational and Environmental Medicine. 2021;63(2):e80

[28] MacIntyre CR, Chughtai AA. Facemasks for the prevention of infection in healthcare and community settings. Bmj. 2015;350.

[29] Anedda J, Ferreli C, Rongioletti F, Atzori L. Changing gears: Medical gloves in the era of coronavirus disease 2019 pandemic. Clinics in Dermatology. 2020.

[30] Garrido-Molina JM, Márquez-Hernández VV, AlcaydeGarcía A, Ferreras-Morales CA, García-Viola A, AguileraManrique G, et al. Disinfection of gloved hands during the COVID-19 pandemic. Journal of Hospital Infection. 2021;107:5-11

[31] Korth J, Wilde B, Dolff S, Anastasiou OE, Krawczyk A, Jahn M, et al. SARS-CoV-2-specific antibody detection in healthcare workers in Germany with direct contact to COVID-19 patients. Journal of Clinical Virology. 2020;128:104437.

[32] Brehm TT, Schwinge D, Lampalzer S, Schlicker V, Küchen J, Thompson M, et al. Seroprevalence of SARS-CoV-2 antibodies among hospital workers in a German tertiary care center: A sequential follow-up study. International Journal of Hygiene and Environmental Health. 2021;232:113671.

[33] Xu X, Sun J, Nie S, Li H, Kong Y, Liang M, et al. Seroprevalence of immunoglobulin $\mathrm{M}$ and $\mathrm{G}$ antibodies against SARS-CoV-2 in China. Nature Medicine. 2020;26(8):11935.

[34] Steensels D, Oris E, Coninx L, Nuyens D, Delforge M-L, Vermeersch P, et al. Hospital-wide SARS-CoV-2 antibody screening in 3056 staff in a tertiary center in Belgium. Jama. 2020;324(2):195-7.

[35] Sims MD, Maine GN, Childers KL, Podolsky RH, Voss DR, Berkiw-Scenna N, et al. COVID-19 seropositivity and asymptomatic rates in healthcare workers are associated with job function and masking. Clinical infectious diseases: an official publication of the Infectious Diseases Society of America. 2020.

[36] Keeley AJ, Evans C, Colton H, Ankcorn M, Cope A, Bennett T, et al. Roll-out of SARS-CoV-2 testing for healthcare workers at a large NHS Foundation Trust in the United Kingdom, March 2020. Eurosurveillance. 2020;25(14):2000433.

[37] Rudberg A-S, Havervall S, Månberg A, Falk AJ, Aguilera $\mathrm{K}, \mathrm{Ng} \mathrm{H}$, et al. SARS-CoV-2 exposure, symptoms and seroprevalence in healthcare workers in Sweden. Nature Communications. 2020;11(1):1-8.

[38] Thomas JP, Srinivasan A, Wickramarachchi CS, Dhesi PK, Hung YM, Kamath AV. Evaluating the national PPE guidance for NHS healthcare workers during the COVID-19 pandemic. Clinical Medicine. 2020;20(3):242.

[39] Hoernke K, Djellouli N, Andrews L, Lewis-Jackson S, Manby L, Martin S, et al. Frontline healthcare workers' experiences with personal protective equipment during the COVID-19 pandemic in the UK: a rapid qualitative appraisal. BMJ Open. 2021;11(1):e046199.

[40] Long Q-X, Liu B-Z, Deng H-J, Wu G-C, Deng K, Chen $\mathrm{Y}-\mathrm{K}$, et al. Antibody responses to SARS-CoV-2 in patients with COVID-19. Nature Medicine. 2020;26(6):845-8.

[41] Self WH, Tenforde MW, Stubblefield WB, Feldstein LR, Steingrub JS, Shapiro NI, et al. Seroprevalence of SARS-CoV-2 among frontline health care personnel in a multistate hospital network-13 academic medical centers, April-June 2020. 2020. 